\title{
Streaming Video and Podcasting Enhancements in the Post-secondary Classroom
}

\author{
Linda Chmiliar \\ Athabasca University, Canada
}

\begin{abstract}
The use of online learning enhancements is becoming more popular in post-secondary instruction, and certainly distance education and lecture based institutions are exploring the use of $e$ learning strategies. Unfortunately, there is very little available research in the literature that examines the use of streaming video and podcasting as enhancements to in class post-secondary instruction. This paper explores the use of short streaming video clips and audio podcasts with students enrolled in a lecture based pre-service teacher education class. Seven short video clips were developed that extended the classroom lecture material. The clips consisted of Power Point slides enhanced with voice, diagrams, and pictures. The clips were also made available to the students as audio only podcasts. Students voluntarily chose whether or not to view the video clips or listen to the audio only podcasts. A supporting study guide and workbook were also provided online. Student perspectives on the use of these online enhancements were gathered during the course feedback process. The benefits and pitfalls of using streaming video/podcasts as part of postsecondary instruction are discussed.
\end{abstract}

\section{Introduction}

Despite the increased availability and emphasis on e-learning tools in post-secondary education, there is limited evidence of the impact of these tools on individual learning and student performance, or information on student perspectives regarding the usefulness of these tools. Streaming video and podcasting and are e-learning tools that have the potential to offer benefits to post-secondary students. They can be used in many ways: to capture in class lectures with audio or video recordings so students can view or listen them at a later time, to extend student learning by providing additional content in a different format, or to provide information and activities that deepen student understanding of the material by involving them in the creative process. The use of streaming video and podcasting may also provide opportunities to deliver course content to students in a way that is engaging and interactive. This project looked at the use of short streaming video clips and podcasts with pre-service education students in an introductory educational psychology course. The video clips and podcasts were offered as e-learning enhancements to a lecture based classroom learning experience. Support was also provided with an online study guide and workbook. The study guide indicated to the students when they should be participating in the video clips/podcasts and provided learning objectives. Some of the video clips/podcasts also had workbook pages that accompanied them, where students were provided with activities to complete as they participated in the video clips/podcasts. As part of the course evaluation, students provided feedback on whether or not they participated in the online environment, which online components they used, how they used the components, and whether or not they found the components to be useful in their learning.

\section{Research Background}

The background information is divided into two parts. The first part discusses the literature found related to the use of podcasts as learning enhancements in post-secondary education. The second part examines the use of streaming video.

Podcasts can be used in the lecture environment to enhance the student experience in a number of ways [1]. There is some discussion in the literature as to the usefulness of podcasting in post-secondary education. However, relatively few studies have investigated students' perceptions of podcasting in post-secondary courses [2], and the pedagogical value of podcasting within the traditional lecture format has been raised [3].

Several studies were found that focused on the use of podcasting. Sutton-Brady, Scott, Taylor, Carabetta, and Clark [4] investigated the use of short podcasts to support assessment for postgraduate and undergraduate students. The majority of the students involved in the research indicated that they benefited from the podcasts and liked the flexibility of the podcasts to support their learning. However, most of the students used the podcasting in their traditional study space at home. Luna \& Cullen [5] shared the results of an exploratory study that looked at graduate student perceptions of podcasting access and usability. Students reported that listening to the podcasts enhanced their perceived understanding of the subject, with $76 \%$ indicating that the podcast enhanced or clarified their understanding of the material. However, $12 \%$ of the students had a neutral response to the podcasts and $12 \%$ of the students indicated that the podcasts were of no value and unimportant in their learning. Jalali, Leddy, Gauthier, Sun, Hincke, and Carnegie [6] looked at 
the use of podcasting as an educational tool with 40 first-year medical students. On a questionnaire, students indicated that they liked the podcasting to review anatomy lecture material. They liked the short length of the podcast, the clarity of the content, and the self-paced learning. The combination of auditory podcasts and visual PowerPoint was identified as facilitating learning. Van Zanten, Somogyi and Curro [3] explored how students interacted with short 3-5 minute podcasts as well as full lecture podcasts. They found that students valued the full lecture podcasts as highly as the short summary podcasts. Fernandez, Simo, and Sallan [7] looked at the use of 13 podcasts in an undergraduate course in information systems management. They reported a number of findings including that fact that the use of podcasting allowed for a diverse range of student skills and learning strategies. They concluded that podcasting is a powerful tool as a complement to the traditional course based resources. Walls, Kucsera, Walker, Acee, McVaugh, Robinson [2] reported positive results with the use of podcasts, but found that students had concerns about the use of podcasting for repetitive or supplemental educational experiences. However, with exposure to the podcasts, students could overcome their reluctance to participate with this medium.

Overall, the majority of the studies reported positive outcomes with the use of podcasts as enhancements in courses. The positive aspects included the flexibility of the medium, the ability to self-pace viewing, enhanced understanding of the material covered, and the ability to learn in several ways which facilitated student learning styles. However, some challenges also emerged. Several studies reported that some students found that the podcasts did not add to their learning experience and concerns were expressed about repetitive activities and student workload.

Video streaming in education is becoming more common and can bring a course alive by providing students with visual and auditory information to learn complex concepts and procedures. Video streaming can augment text-based materials and stimulate interest in the subject matter [8]. Video is 'streamed' over the internet and students access the content by clicking on a hyperlink within a webpage. There are few documented and evaluated examples of the use of streaming video in post- secondary education environments [9]. Romanov [10] looked at the use of six learning modules with integrated video clips in WebCT with medical students. Sixty eight percent of the students watched the videos, and video watchers were more active in the course. Videowatching was also associated with a better course grades. Evans [11] examined student reactions to a set of screencasts introduced to accompany embryology lectures. Five mini-lecture screencasts accompanied by a review quiz were incorporated into a lecture course. Data showed that the screencasts were accessed by many of the students and students accessed the screencasts at many different times. Students indicated that they found that the screencasts were useful to their learning and there was some data to indicate that the screencasts had a positive effect on student outcomes. Cofield [12] conducted an assessment of streaming video in web-based instruction. Sixty-nine college students reported that they felt that the streaming video clips acted as learning reinforcement and that the clips supported their attention and learning styles. Kukulska-Hulme, Foster-Jones, Jelfs, Mallett, and Holland [10] investigated the usefulness of digital video applications in distance learning. They reported concerns about added student workload, technical issues, and the quality of the video related to bandwidth. Nicholson and Nicholson [13] reported on student and faculty perception on streaming video included in an introductory management information systems course. Through a qualitative survey they found benefits to the students in terms of greater satisfaction with the learning process, a greater understanding of the course material, and a reported reduction in the amount of effort required to finish the course. They also reported instructor satisfaction with the video due to a reduction in student inquiries about the course material covered, and a decrease in preparation time in subsequent versions of the course. Green, Voegeli, Harrison, Philips, Knowles, Weaver, and Shepard [14] looked at the use of streaming video to support student nurses in a first year course. Their results indicated that $32 \%$ of the students found access to the videos easy and $59 \%$ of the students enjoyed viewing the videos.

Overall, the use of video streaming produced positive results in the studies reviewed, similar to the studies examining the use of podcasting. Students enjoyed viewing the videos and felt that the videos facilitated their attention and learning styles. Several studies evidenced positive student outcomes including higher grades. However, technical issues arose, as did issues with video quality related to band width. Concerns with student workload also emerged.

\section{Implementation}

Given the distinct shortage of information and literature on the development and use of video streaming and podcasting in the post-secondary environment, it was decided to develop and test these tools in a classroom environment. Two hundred and eighty three students enrolled in a 13 week lecture based teacher preparation course were offered the opportunity to participate in e-learning activities as part of their course. 
Seven short video clips were developed by the course professor using Camtasia software. The video clips consisted of information on PowerPoint slides with visual enhancements and voice. The clips were developed to cover and extend content covered in the textbook and lectures, and all were less than 20 minutes in length. In addition to the streaming video clips, an online study guide was developed to support learning in the course and the use of the streaming video clips. The study guide indicated to the students when they should be viewing the streaming video clips and provided learning objectives for the clips. An online workbook was also developed to guide student viewing and note-taking during viewing. These pages could be downloaded prior to viewing. The workbook pages were set up so that the student would have to structure and organize the information that was provided in the streaming video clips/podcasts. Several of the workbook pages also included activities to complete. The study guide also outlined the use of the workbook. The students were informed at the beginning of the course of the availability of the online learning tools and it was indicated that a number test items on the 3 multiple choice exams during the course would be based on the content in the streaming video clips/podcasts. Reminders as to the use of the streaming video clips/podcasts were also provided during lecture time.

A considerable amount of time was spent learning how to use the software and produce video of reasonable quality. Once the clips were complete, they were offered to the students on a course webpage as steaming video clips or as audio only podcasts. The students were able to choose the medium that they preferred.

Feedback on participation was gathered as part of the course feedback process at the end of the course. Students provided feedback on the following questions:

Did they participate in the online environment?

How often did they participate online?

Which online components did they use?

How did they use the online components?

How often did they use the components?

Which components did they find to be useful in their learning?

Why were those components useful?

\section{Results}

One hundred and eighty nine of the two hundred and eighty three students enrolled in the class chose to provide feedback at the end of the course on the course evaluation. Of these 189 students, 160 were female and 29 were male. The majority of the students were in the 18 to 25 year old age group who had completed 1 to 3 years of post-secondary instruction. Of the 189 students providing feedback, 161 indicated that they chose to participate in the elearning activities. The remainder did not participate, indicating that they did not have the time or the inclination to participate. The majority of the students participated in the e-learning activities once or twice and then quit. Twenty-eight percent of the students participated a number of times and 19\% participated throughout the course. Seventy-one percent of the students who participated in the streaming video indicated that they found it very or somewhat useful in their studying. Twenty-nine percent indicated that the video was not very, or not at all useful to them. The majority of the students participated in the video clips by viewing the video only, but $24 \%$ reported that they used the audio only option. A laptop was the most common hardware used to access the podcasts, with $12 \%$ reporting that they used a desktop computer, and only .5\% reporting the use of an MP3 or other portable device to access the content.

There were a number of opportunities in the course evaluation for students to respond to open ended questions regarding their participation in the elearning content. Many of the comments were very positive. The most common comment was that students liked the ability to view or listen to the content at their leisure and appreciated the ability to start and stop the video when necessary. Another common theme was that many students appreciated receiving content in another mode. One student wrote, "I found that I really like the podcasts. I did not enjoy that it was more content to attend to in the course, but I found out that I was quite an auditory learner..." Several students reported that the podcasts were useful if they missed a concept in class or needed a refresher for an exam. One student indicated that she did not use the e-learning tools for the first exam, but when she did for the second exam her "mark went up by 24\%." A couple of the students that used a mobile device to access the content indicated they "enjoyed being able to relisten to things while walking to school."

A number of drawbacks were also reported. The most common complaint was that the e-learning activities felt like extra work. A few students indicated that they did not have any time for elearning activities and if they did take the time, they wanted the content directly tested. Several students commented that they like and prefer the lecture environment or just reading from the text to viewing video content. One student reported that she felt that it was "not easy to learn on my own" and that was why she attended lectures. There were several comments regarding technical difficulties such as having difficulties accessing the content on their computer at home, difficulties logging on, and that "online material is a hassle and not reliable and too 
time consuming." One student even indicated that she did not participate because she could not figure out where to go to access the content, and gave up. There were also comments that the videos were repetitive, not as interesting as it could be, and that in some cases the content overlapped with lectures.

\section{Discussion}

Overall, there were many similarities between the data collected and the data reported in the literature. In the present study, students indicated that they found access to the e-learning material, and specifically the video clips, to be helpful in their learning. This was similar to the results of SuttonBrady, Scott, Taylor, Carabetta, and Clark [4], Jalali, Leddy, Gauthier, Sun, Hincke, Carnegie [6], Nicholson and Nicholson [13], and Luna and Cullen [5].

Students in this study reported that they liked increased learner control and the flexibility to access, start, and stop video as desired when using the streaming video clips/podcasts. This finding is similar to that of Jalali, Leddy, Gauthier, Sun, Hincke, and Carnegie [6] where students reported that they liked the self-paced learning. SuttonBrady, Scott, Taylor, Carabetta, and Clark [4] also reported that students liked the flexibility of the podcasts to support their learning.

Many of the students in the present study also appreciated having access to learning material in a different mode for learning and reviewing. Fernandez, Simo, and Sallan [7] found that the use of podcasting allowed for a diverse range of student skills and learning styles. This was also evidenced by Cofield [12], who found that students indicated that the streaming video clips supported their attention and learning styles.

Several students in the present study indicated in their comments that in their perspective, their participation in online learning activities resulted in better grades. Video-watching was associated with better course grades in the study by Romanov [10]. Evans [11] also found that there was data to indicate that screencasts had a positive effect on student outcomes. This could be the result of the enhancement or clarification of the material in the streaming video clips/podcasts as evidenced in the data collected by Luna and Cullen [5].

Sutton-Brady, Scott, Taylor, Carabetta, and Clark [4] found that most of the students participating in their study used the podcasting in their traditional study space at home. In the present study, the majority of the students reported using a lap top to access the streaming video clips/podcasts rather than a desk top computer. However, it is not known if they were using their lap tops in their traditional study space in their homes, or if they were using the lap tops as a mobile device. A minimal number of students in the present study reported downloading the streaming video clips/podcasts onto mobile devices such as an MP3 player despite the ease of using this type of device with this kind of content. It is surprising that with the access to podcasts that can be downloaded onto mobile devices, that so few students actually exercised this option. So many students in this age demographic almost live on their mobile devices, but it appears that using the mobile device for learning purposes is not something that they consider. Perhaps students do not want to use their mobile devices that they use for pleasure and socialization for academic learning activities. Perhaps students are not aware of their ability to download academic materials onto their personal devices. Direction and support in this regard might encourage students to use their mobile tools for learning purposes.

Not all students in this study chose to participate in the online learning enhancements and a number of students indicated that the streaming video clips/podcasts were not useful to their learning. This was similar to the results presented by Luna and Cullen [5] where $24 \%$ of the participants had a neutral response or negative response to the value of podcasts in their learning. There are likely students in every post-secondary classroom that are not interested in participating in "extra" activities to improve their learning, for whom the most direct track to course completion and grade is satisfactory. These may also be students who have well-rehearsed and effective learning strategies who do not require extra support.

Technical issues with student access to e-learning content appeared to be an issue for some students in the present study, even with a younger age demographic. Similar concerns were also expressed by Kukulska-Hulme, Foster-Jones, Jelfs, Mallett, and Holland [9], who reported technical issues, and difficulties with the quality of the video related to bandwidth. Computer literacy and student motivation and ability to problem solve technical difficulties should not be assumed. Many students have access to current educational technologies and have the skills to access and use online materials. Other students may have access to technology, but the technology may not be current and lack the memory capabilities, program updates, and bandwidth that make access seamless for students. There may also still be students whose computer literacy is still at a rudimentary level.

The time commitment for students was a significant issue in the perspective of a number of students in this study. Students may have concerns about the use of podcasting for repetitive or supplemental educational experiences as indicated in the study by Walls, Kucsera, Walker, Acee, McVaugh, and Robinson [2]. When using 
supplemental streaming video clips/podcasts, it may be necessary to build time into the course schedule for students to access the content. Students may also need to see the direct link between the streaming video clips/podcasts to course objectives, assessment strategies, and feedback in order to be motivated to participate in e-learning activities.

\section{Conclusion}

The use of streaming video/podcasting in postsecondary environments offers considerable opportunities for teaching staff to develop engaging learning activities for students. Streaming video /podcasting offers students the opportunity to learn course content in different ways in their home environment, or on the move with mobile devices. There is a growing body of evidence in the literature to indicate that many students find streaming video /podcasts to be useful in their learning. They value the flexibility of these tools and the fact that the presentation of course content using these types of media does accommodate different learning styles and rates. There is also evidence that the use of these online learning enhancements can have an impact on student performance. However, there are a number of issues that need to be addressed when implementing these strategies. Students are concerned about the time commitment involved in using these tools, some students may lack access to the necessary computer technologies, and some students may still not have the computer literacy skills to access the materials in an effective and timely manner. Finally, although many students have indicated in this and other studies in the literature that they have benefitted from the use of streaming video/podcasting, there may be students who do not wish to engage in this kind of learning. These students may need to have the option to not participate, or may require additional encouragement to explore the learning options and opportunities these tools afford.

\section{Acknowledgements}

The author would like to thank the many students who thoughtfully provided feedback on their online learning experiences.

\section{References}

[1] O. McGarr, "A review of podcasting in Higher Education: Its Influence on the Traditional Lecture", Australasian Journal of Educational Technology, 2009, pp. 309-321.

[2] S. Walls, J. Kucsera, J. Walker, T. Acee, N. McVaugh, and D. Robinson, D. "Podcasting in Education: Are
Students as Ready and eager as We Think They are?” Computers \& Education, 2010, pp. 371-378.

[3] R.Van Zanten, S. Somogyi, and G. Curro, "Purpose and Preference in Educational Podcasting”, British Journal of Educational Technology, 2012, pp. 130-138.

[4] C. Sutton-Brady, K. Scott, L. Taylor, G. Carabetta, and S. Clark, S. "The Value of Using Short-Format Podcasts to Enhance Learning and Teaching", ALT-J: Research in Learning Technology, 2009, pp. 219-232.

[5] G. Luna, and D. Cullen, "Podcasting as Complement to Graduate Teaching: Does it Accommodate Adult Learning Theories?”, International Journal of Teaching and Learning in Higher Education, 2011, pp. 40-47.

[6] A. Jalali, J. Leddy, M. Gauthier, R. Sun, M. Hincke, and J.Carnegie, "Use of Podcasting as an Innovative Asynchronous E-Learning Tool for Students”. US-China Education Review, 2011, pp. 741-748.

[7] V. Fernandez, P. Simo, and J. Sallan, "Podcasting: A New Technological Tool to Facilitate Good Practice in Higher Education”, Computers in Education, 2009, pp. 385-392.

[8] T. Hartsell, and S. Chi-Yin Yuen, "Video Streaming in Online Learning”, AACE Journal, 2006, pp. 31-43.

[9] A. Kukulsak-Hulme, J. Foster-Jones, A. Jelfs, E. Mallett, and D. Holland, "Investigating Digital Video Applications in Distance Learning”, Journal of Educational Media, 2004, pp. 125-137.

[10] K. Romanov, "Do Medical Students Watch Video Clips in E-Learning and do they Facilitate Learning?”, Medical Teacher, 2007, pp. 490-494.

[11] D. Evans, "Using Embryology Screencasts: A Useful Addition to the Student Learning Experience?", Anatomical Sciences Education, 2011, pp. 57-63.

[12] J. Colfield, "An Assessment of Streaming Video in Web-based Instruction”, Paper presented at the Annual Meeting of the mid-South Research Association, 2002, http://www.eric.ed.gove/contentdelivery/servlet/ERICServ let?accn0=ED471348

[13] J. Nicholson, and D. Nicholson, A" Stream Runs thought IT: Using Streaming Video to Teach Information Technology", Campus-Wide Information Systems, 2010, pp. 17-24.

[14] S. Green, D. Voegeli, M. Harrison, J. Philips, J. Knowles, M. Weaver, and K. Shepard, "Evaluating the Use of Streaming Video to Support Student Learning in a First-Year Life Sciences Course for Student Nurses”, Nurse Education Today, 2003, pp. 255-261. 